\title{
Expression of Sucrose Transporters from Vitis vinifera Confer High Yield and Enhances Drought Resistance in Arabidopsis
}

\author{
Yumeng Cai ${ }^{1,2}{ }^{,}$Jing Yan ${ }^{1}$, Wenrui Tu ${ }^{1}$, Zhefang Deng ${ }^{1}$, Wenjie Dong ${ }^{1}$, Han Gao ${ }^{1}$, Jinxu Xu ${ }^{1}$, \\ Nan Zhang ${ }^{1}$, Ling Yin ${ }^{3}$, Qingyong Meng ${ }^{4,5}$ - and Yali Zhang ${ }^{1, *}$ \\ 1 Beijing Advanced Innovation Center for Food Nutrition and Human Health, College of Food Science and \\ Nutritional Engineering, China Agricultural University, Beijing 100083, China; \\ caiyumeng917@163.com (Y.C.); yanjingjing1618@163.com (J.Y.); twr6637@163.com (W.T.); \\ 531128763@163.com (Z.D.); dongwenjie1134@163.com (W.D.); fadaiclub@126.com (H.G.); \\ xujinxujason@gmail.com (J.X.); zhangnancau@163.com (N.Z.) \\ 2 Tianjin Key Laboratory of Crop Genetics and Breeding, Crops Research Institute, Tianjin Academy of \\ Agricultural Sciences, Tianjin 300384, China \\ 3 Guangxi Crop Genetic Improvement and Biotechnology Key Lab, Guangxi Academy of Agricultural \\ Sciences, Nanning 530007, China; yinling1985@gmail.com \\ 4 Beijing Advanced Innovation Center for Food Nutrition and Human Health, College of Biological Science, \\ China Agricultural University, Beijing 100193, China; qymeng@cau.edu.cn \\ 5 The State Key Laboratory for Agrobiotechnology, College of Biological Sciences, China Agricultural \\ University, Beijing 100193, China \\ * Correspondence: zhangyali@cau.edu.cn; Tel.: +86-010-62737465
}

Received: 1 February 2020; Accepted: 5 March 2020; Published: 9 April 2020

\begin{abstract}
Sucrose is the predominant form of sugar transported from photosynthetic (source) to non-photosynthetic (sink) organs in higher plants relying on the transporting function of sucrose transporters (SUTs or SUCs). Many SUTs have been identified and characterized in both monocots and dicots. However, the function of sucrose transporters (SUTs or SUCs) from Vitis is not clear. As the world's most planted grape species, Vitis vinifera owns three sucrose transport activity verified SUTs. In this study, we constructed three kinds of VvSUC (Vitis vinifera SUC)-overexpressing transgenic Arabidopsis. VvSUC-overexpressing transgenic Arabidopsis was cultured on sucrose-supplemented medium. VvSUC11- and VvSUC12-overexpressing lines had similar thrived growth phenotypes, whereas the size and number of leaves and roots from VvSUC27-overexpressing lines were reduced compared with that of WT. When plants were cultured in soil, all SUT transgenic seedlings produced more number of leaves and siliques, resulting in higher yield (38.6\% for VvSUC12-transformants) than that of WT. Besides, VvSUC27-transformants and VvSUC11-transformants enhanced drought resistance in Arabidopsis, providing a promising target for crop improvement
\end{abstract}

Keywords: Vitis vinifera; VvSUC; overexpression; Arabidopsis; srong phenotype; high production; drought resistance

\section{Introduction}

Carbon fixation occurs in photosynthetic (source) organs, such as mature leaves. Carbon fixed in excess during the day can be exported as sucrose to the non-photosynthetically heterotrophic (sink) organs, such as roots, developing leaves, reproductive organs, seeds, the shoot apex, tubers and meristems [1]. Therefore, the function of sucrose transporters (SUTs or SUCs) in the translocation of sucrose is indispensable for plant development. Since the discovery of spinach SoSUT1 [2], several 
other SUTs have been identified and characterized in both monocots and dicots [3-7]. However, not all SUT family members are well characterized and our understanding on the function of SUTs in Vitis is limited.

Phylogenetic analysis has revealed that there are five independent SUT subfamilies that make up the type SUT1-SUT5 clades [8], and dicotyledonous transporters belong to the SUT1, SUT2/SUC3, and SUT4 subfamilies. Most members of the SUT1 clade are high affinity Suc/proton cotransporters that are mostly located on the plasma membranes of the sieve element (SE) [9-11], companion cells (CC) [8], or both cell types [12]. SUT1 is mainly expressed in sucrose-exporting source leaves [11]. Similarly, LeSUT1 is the main phloem loader of sucrose in Solanaceae [10].

Most members of the SUT2/SUC3 and SUT4 subfamilies have been reported to be low-affinity sucrose transporters. The SUT2/SUC3 clades are structurally different from other SUT clades as they have extended domains at their N-termini and elongated central cytoplasmic loops [13]. The SUT2/SUC3 transporters are localized to SE plasma membranes $[10,11,14,15]$, except ZmSUT2, which is on the vacuolar membrane. ZmSUT2 can remobilize sucrose from the vacuole, which contributes to maize development and agronomic yield [16]. SUT2/SUC3 is reported to be expressed predominantly in the sink organs, such as sink leaves, stems, and fruits, whereas LeSUT2 and AtSUC3 (AtSUT2) are colocalized in the SEs [11].

The SUT4 subfamilies have been mostly identified in diverse subcellular localizations and with diverse functions; for example, AtSUT4, LeSUT4, and StSUT4 are localized to enucleated SEs in plants [17]. StSUT4 is located on the plasma membrane, mainly in the sink leaves, of young potato plantlets and at the perinuclear ring, which could affect flowering, tuberization, shade-avoidance response, circadian-regulated genes, and ethylene production $[7,18]$. AtSUT4 has been identified in the vacuole, suggesting that it is involved in the transport and vacuolar storage of sucrose [19].

Multiple SUT isoforms are responsible for loading phloem, transporting and unloading sucrose; however, the SUT clades in vine species have not been well characterized with respect to SUT functional diversity. Vitis as a genus has about 60 vining species. Vitaceae species are used as medicinal herbs and wine making worldwide [20]. The type and concentration of sugars and acids in grapes contribute to the organoleptic quality of the berries, the flavor and stability of wine [21]. Sucrose is the predominant form of sugar transportation in grapevine; therefore, the function of Vitis vinifera SUCs (VvSUCs) are important. Although the three functional SUTs-VvSUC11, VvSUC12, and VvSUC27-have been identified in grape berries [22], differences in the function of these SUTs in $V$. vinifera is not well understood. As a member of the SUT1 subfamily, VvSUC27 has been described as a low-affinity/high-capacity SUT, VvSUC11 has been characterized as a member of the traditional low-affinity SUT4 subfamily, while VvSUC12 belongs to the SUT2/SUC3 subfamily [23-26]. Previously, we have elucidated that VvSUC27 is located on the plasma membrane and negatively correlated with sugar accumulation, which indicates its important role in plant development [27]. In this study, the functions of SUTs were further systematically evaluated using transgenic Arabidopsis thaliana lines.

\section{Results}

\subsection{Amino Acids Sequences Analysis of VvSUC Proteins}

We initially studied the phylogenetic relationships between SUT sequences in Arabidopsis thaliana, Lycopersicon esculentum, and Vitis vinifera "Thompson Seedless" (Figure S1). The SUT sequences in V. vinifera can be classified into three broad classes and share $40.95 \%$ to $51.61 \%$ similarity between each other (Table S1).

\subsection{SUT Overexpression Alters Morphological Phenotypes and the Germination Rate of Arabidopsis Seeds}

To further characterize the functional differences between the three SUTs, homozygous $\mathrm{T}_{3}$ VvSUC-overexpressing (OE) lines of Arabidopsis were produced and verified by PCR (Figure S2A-C) and RT-qPCR (Figure S2D). The length and width decide the size of the seed, the length reflects the 
length of the hypocotyl-root axis (major axis), and the width is essentially the sum of the cotyledon and hypocotyl widths (minor axis). Therefore, the size and shape of the exalbuminous seed were determined by the embryo structure and how the embryo folded within [28]. Scanning electron micrographs showed that the embryo folded within VvSUC11- and VvSUC12-OE seeds (Figure 1B,C) were basically normal compared with that of wild type (WT) seeds (Figure 1A), whereas VvSUC27-OE seeds had significantly deeper folded embryo (Figure 1D). Size differences between the embryos of VvSUC-OE and WT seeds are compared in Figure 1E. There was significant difference between the length (i.e., the hypocotyl root major axis) of VvSUC-OE and WT seeds; VvSUC-OE seeds were shorter than WT seeds and VvSUC11-OE seeds were the longest among the VvSUC-OE seeds analyzed. The length of VvSUC12- and VvSUC27-OE seeds was similar to each other. Among all the seeds evaluated, in the matter of width, VvSUC12-OE seeds were the largest (e.g., cotyledon and hypocotyl widths) and VvSUC27-OE seeds were the smallest. The VvSUC27-OE seeds were the smallest and WT seeds were the largest. The inhibitory effects of exogenous sucrose on germination in the different VvSUC-OE lines indicated that high sucrose concentrations resulted in a lower germination rate of all the seeds, although this inhibition attenuated over time (Figure 1F). The inhibition rate of each VvSUC-OE line was compared with that of WT on $6 \%$ sucrose-supplemented medium, and the results indicated that VvSUC11-OE lines had a similar inhibition rate, while VvSUC12-OE and VvSUC27-OE lines had weaker and stronger germination inhibition rates than that of WT, respectively (Figure $1 G$ ).
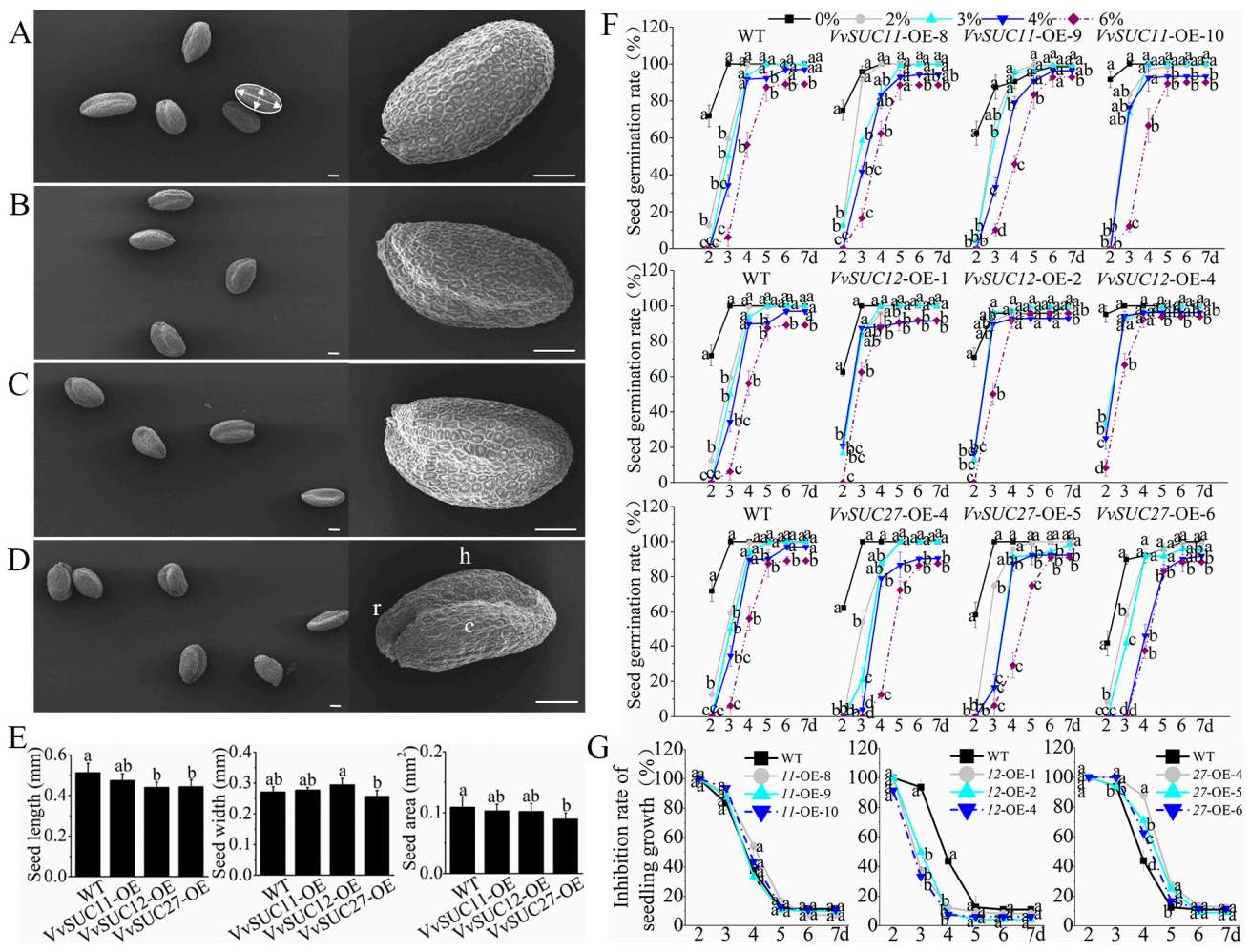

Figure 1. Morphological phenotypes of seeds and the effects of sucrose concentration on the seed germination of Vitis vinifera sucrose transporter (VvSUC)-overexpressing (OE) Arabidopsis. (A) to (D) morphological phenotypes were observed by scanning electron microscopy in the (A) wild type (WT), (B) VvSUC11-, (C) VvSUC12-, and (D) VvSUC27-OE lines Arabidopsis seeds. Magnification of the seeds for each transgenic line (right) highlight changes in seed morphology; c, cotyledon; h, hypocotyl; and $\mathrm{r}$, embryonic root or radicle. Bars $=100 \mu \mathrm{m}$. The white arrows indicate the major and minor axes of a successfully segmented seed. (E) The seed length, width, and area were compared using scanning electron microscopy images. The seed length and width were measured as major and minor axes (white arrows in A), respectively. For seed area, a custom algorithm for ellipse area determined the boundary or contour of the seed (white circle in A). For all lines, 20 seeds from each variety were counted and separated (segmented), and the seeds that were touching each other or a piece of debris were omitted 
from the analysis. Data are expressed as mean $\pm S D(n=20)$. (F) Seed germination rate of VvSUC-OE lines grown on different sucrose concentrations. Sterilized seeds were placed on Murashige and Skoog (MS) media containing $0 \%, 3 \%, 4 \%$, and $6 \%(w / v)$ sucrose. Seed germination was defined and measured as radicle protrusion. Each culture vessel contained 80 seeds. Data are expressed as mean $\pm \operatorname{SD}(n=3)$. (G) Inhibition rate of seed germination was measured in VvSUC-OE seeds and WT grown on MS medium containing $6 \%(w / v)$ sucrose. Different letters indicate significant differences $(P<0.05)$ between each VvSUC-OE seeds and WT, as determined by ANOVA followed by Tukey's test.

\subsection{VvSUC-OE Lines Have Developmental Phenotypes When Grown on Sucrose}

The seeds of the VvSUC-OE lines were sterilized and germinated on Murashige and Skoog (MS) medium supplemented with $0 \%, 3 \%$, and $6 \%$ sucrose under in vitro conditions, and then 4-week-old seedlings were harvested to analyze the influence of sucrose on VvSUC-OE plant growth (Figure 2). There was no significant difference in phenotypes between the VvSUC-OE lines and WT grown on no sucrose-supplemented MS medium (Figure 2A,B). The VvSUC11- and VvSUC12-OE lines had a stronger developmental phenotype (more number,longer and larger leaves) than that of WT on both sucrose-supplemented MS media (Figure 2A,B). As the sucrose concentration increased to $6 \%$, the roots of VvSUC11- and VvSUC12-OE lines had a more number of lateral roots, but their root length and leaf number were unchanged; however, the leaf length and area decreased in these lines compared with themselves on medium with 3\% sucrose (Figure 2A,B). In VvSUC27-OE lines, the VvSUC27-OE lines showed the shorter leaves and roots, less number of, and smaller leaves on both sucrose-supplemented MS media; besides, the higher sucrose concentration did not influence root length or leaf number, length, and area; however, the leaf color was purple on 6\% sucrose MS medium (Figure 2A,B). It has been reported that sucrose signaling can specifically induce anthocyanin accumulation [29]. Because the purple coloration may indicate the synthesis and accumulation of anthocyanins, we investigated the expression of the main anthocyanin biosynthetic genes [30], AtCHS [31], AtDFR [32], and AtLDOX [33] and found that these genes in the VvSUC27-transformants were induced, especially on $6 \%$ sucrose-supplemented MS medium (Figure 2C).

\subsection{Dry Weight, Soluble Sugar Concentrations, and Endogenous Plant Growth Hormone Levels Were Altered in the VvSUC-OE Lines}

The VvSUC-OE lines had different growth phenotypes after four weeks of cultivation in response to sucrose concentration. The VvSUC11- and VvSUC12-OE lines developed thicker leaves and roots on sucrose-supplemented media than those of WT plants. Although the VvSUC27-OE lines showed inhibited phenotypes, the leaves and roots were thicker than those of WT (data not shown). We measured the dry weight of 4-week-old transformants and found that compared with that of WT, VvSUC11- and VvSUC12-OE lines had significantly improved growth on sucrose-supplemented media. Additionally, we observed increased root, shoot (leaves and stems), and whole plant weight of the VvSUC-OE lines on both $3 \%$ and $6 \%$ sucrose-supplemented media compared with those of WT (Figure 3A). The thicker leaves and roots of the VvSUC27-OE lines did not contribute to increased dry weight; the root, shoot, and whole plant dry weight were significantly less than those of WT. The dry weight ratio was not significantly different between the VvSUC-OE lines and WT on media without sucrose. The dry weight of both VvSUC11- and VvSUC12-OE lines revealed higher root/whole plant ratio, but lower shoot/whole plant ratio, indicating a decreased shoot/root ratio compared with that of WT grown on 3\% and 6\% sucrose-supplemented media. Interestingly, the dry weight of roots or shoots/whole plant ratios of the VvSUC27-OE lines were similar to those of WT; however, the shoot/root ratio was marginally higher in $3 \%$ and $6 \%$ sucrose-supplemented media, indicating a more severe developmental inhibition of root than shoot growth in the VvSUC27-OE lines. 

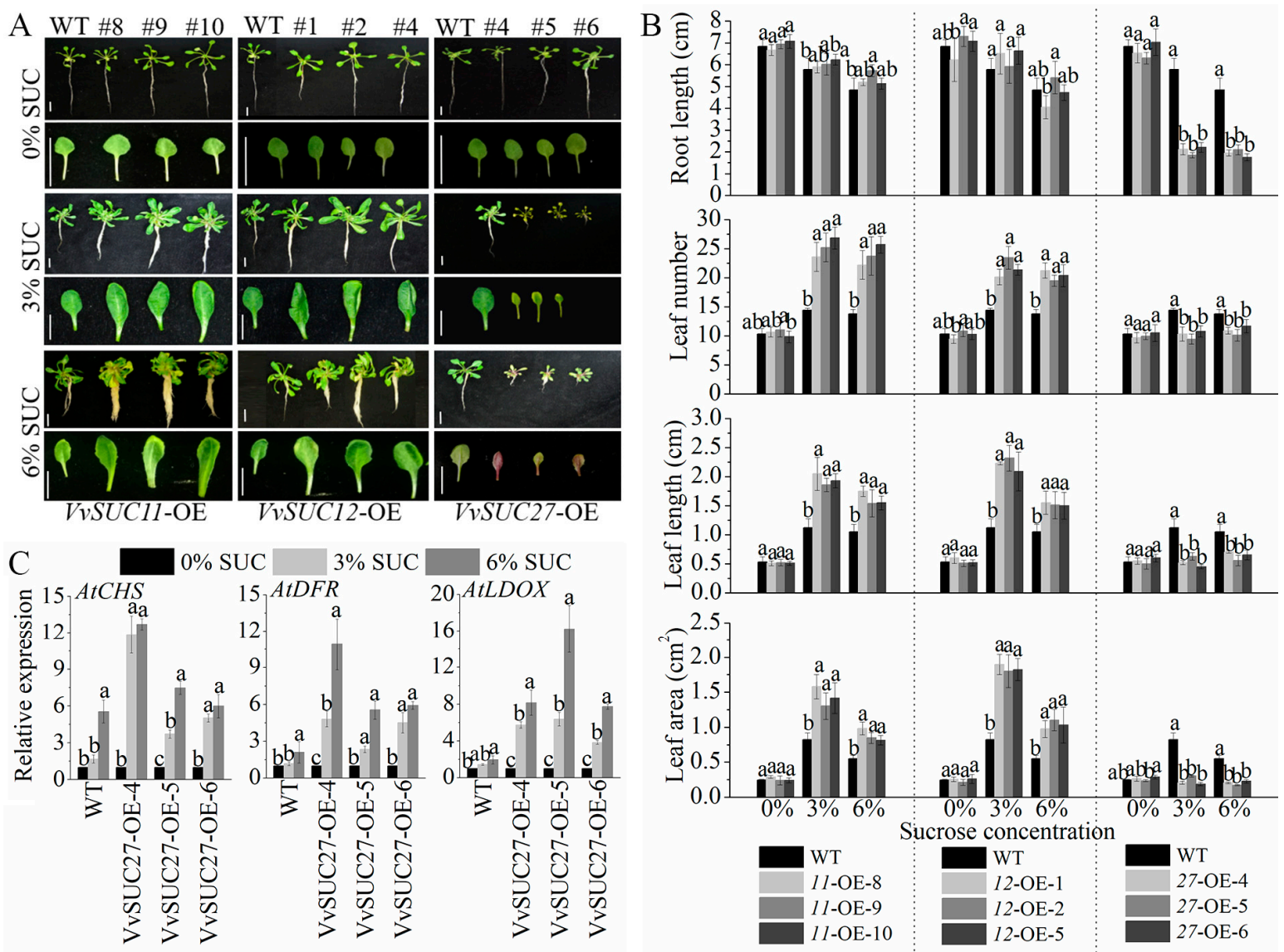

Figure 2. Phenotypes of 4-week-old VvSUC-OE Arabidopsis grown in medium supplemented with different sucrose concentrations. (A) The phenotype differences of whole seedlings and leaves of VvSUC11-, VvSUC12-, and VvSUC27-OE lines, compared with those of WT. Bars $=1 \mathrm{~cm}$. (B) The phenotype differences in the root length, leaf number, leaf length, and leaf area between VvSUC-OE lines and WT. Data are expressed as mean \pm SD $(n=5)$. Different letters indicate significant differences $(P<0.05)$ between each VvSUC-OE line and WT at one sucrose concentration, as determined by ANOVA followed by Tukey's test. (C) The expression levels of AtCHS, AtDFR, and AtLDOX in VvSUC-OE grown on MS media supplemented with $0 \%, 3 \%$, and $6 \%$ sucrose. The cDNA from $4-\mathrm{w}$-old leaves was isolated and tested for the presence of Actin, which served as the internal control for gene expression. Relative gene expression in each VvSUC-OE line was calculated by comparing the expression values from seedlings grown on sucrose-supplemented media with those of the control medium, which was set to one. Data are expressed as mean $\pm \mathrm{SD}(\mathrm{n}=3)$. Different letters indicate significant differences $(P<0.05)$ among different sucrose concentrations, as determined by ANOVA followed by Tukey's test.

We then verified whether soluble sugar concentrations were affected by VvSUC-OE. Compared with that of WT, increased sucrose concentrations could only be observed in the roots of VvSUC11- and VvSUC12-OE lines grown on 3\% and 6\% sucrose-supplemented media (Figure 3B). The soluble sugar concentrations also increased in the roots of VvSUC27-OE lines grown on 3\% sucrose-supplemented medium but decreased in plants grown on $6 \%$ sucrose-supplemented medium; however, the soluble sugar concentration in the shoots was higher than that in WT on both $3 \%$ and $6 \%$ sucrose-supplemented media (Figure 3B).

The endogenous plant growth hormone indole-3-acetic acid (IAA) was also extracted and measured from the seedlings of 4-week-old VvSUC-OE lines (Figure 3C). Compared with that of WT, there was no obvious pattern of IAA accumulation in the VvSUC-OE lines grown on medium without sucrose; however, the VvSUC-OE lines had higher IAA concentration on both 3\% and 6\% sucrose-supplemented media. 

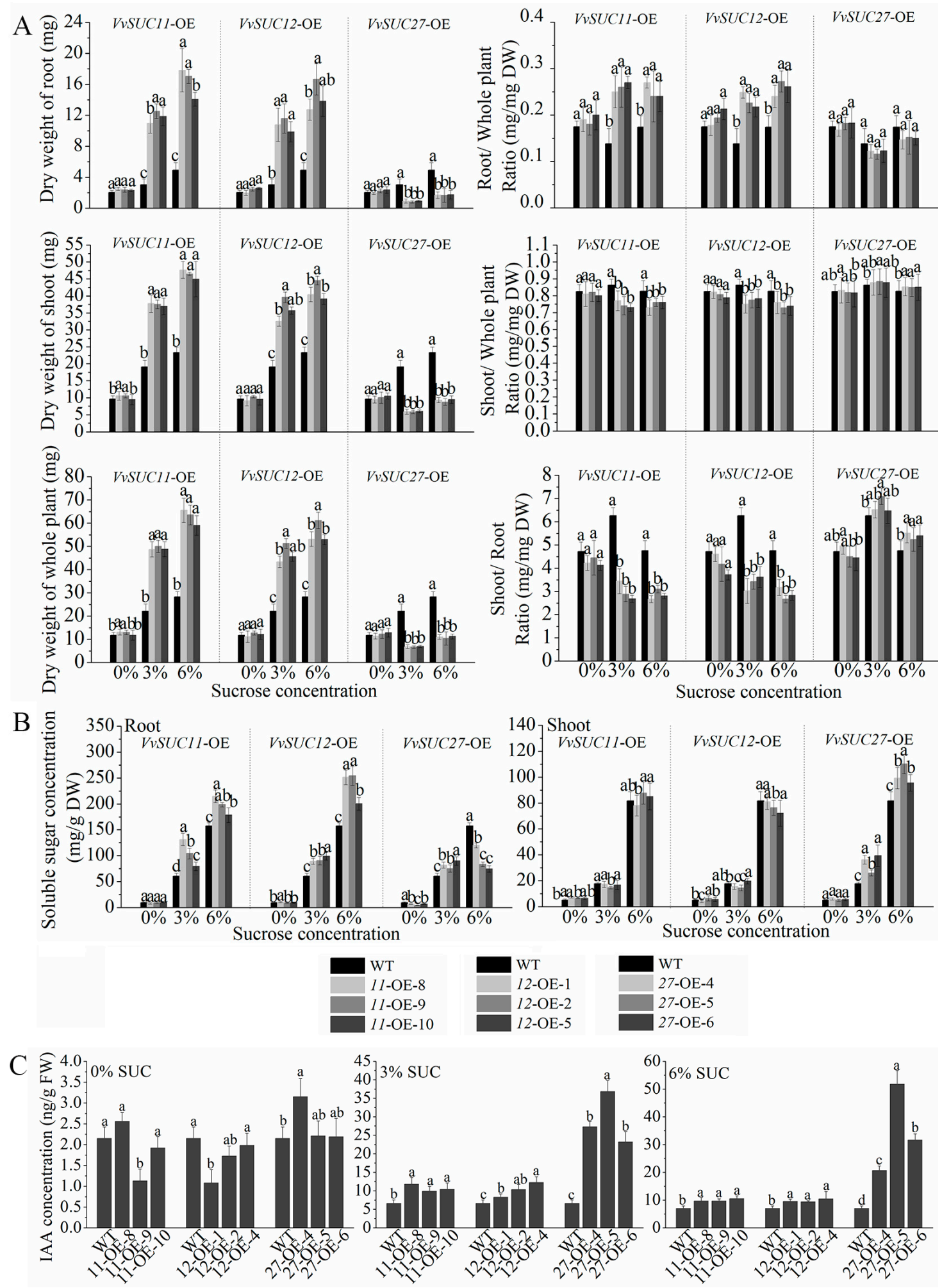

Figure 3. Dry weight, soluble sugar concentration, and endogenous plant auxin levels in 4-week-old VvSUC-OE Arabidopsis grown in media supplemented with different sucrose concentrations. (A) The dry weight of roots, shoots, and whole plants and the dry weight ratios for root/whole plant, shoot/whole plant, and shoot/root of VvSUC11-, VvSUC12-, and VvSUC27-OE lines, respectively. DW = dry weight. (B) The soluble sugar concentrations in the roots and shoots of VvSUC-OE lines. (C) The indole-3-acetic acid (IAA) concentration in the seedlings of VvSUC-OE lines. FW = fresh weight. Data are expressed as mean $\pm \mathrm{SD}(\mathrm{n}=3)$. Different letters indicate significant differences $(P<0.05)$ between each VvSUC-OE line and WT, as determined by ANOVA followed by Tukey's test. 


\subsection{VvSUC-OE Lines Have Developmental Phenotypes When Grown in Soil}

After characterizing the effects of sucrose on VvSUC-OE line growth in vitro, the influence of $V v S U C$ overexpression in transgenic Arabidopsis was analyzed in plants grown under soil culture conditions (Figure 4). Compared with that of WT, all 5-week-old VvSUC-OE lines had enhanced developmental phenotypes, including significantly higher leaf number, length, width, and area, which further confirmed an SUT-dependent effect on plant development (Figure 4A,C). We then used the source leaves to measure sucrose uptake in plants by monitoring the uptake of sucrose- $\left[{ }^{14} \mathrm{C}\right]$. All the VvSUC-OE lines had a higher capacity for sucrose uptake into the source leaves than that of WT (Figure S3). Eight-week-old $V v S U C-O E$ lines were noticeably taller and had more numbers of lateral branches than those of WT plants (Figure 4B,C). The yield of the VvSUC-OE lines was also analyzed (Table 1), although we did not find a significant improvement in seed number per silique of the VvSUC-OE lines; however, significant differences were observed in the silique number between the VvSUC11- and VvSUC12-OE lines with WT. The VvSUC11-, VvSUC12-, and VvSUC27-OE lines had increased number of siliques than that of WT, with VvSUC11- and VvSUC12-OE lines presenting the highest number of siliques produced among the transgenic lines. The higher silique number resulted in a higher yield (mg) per plant in the VvSUC-OE lines than that of WT Arabidopsis plants.

Table 1. Yield of VvSUC-OE Arabidopsis thaliana plants.

\begin{tabular}{|c|c|c|c|}
\hline Line & Seeds Number Per Silique & Silique Number & Yield Per Plant (mg) \\
\hline VvSUC11-OE-8 & $44.71 \pm 0.43^{\mathrm{d}}$ & $402.29 \pm 31.76^{a}$ & $339.97 \pm 15.83^{a, b}$ \\
\hline VvSUC11-OE-9 & $44.29 \pm 0.21^{\mathrm{d}, \mathrm{e}}$ & $350.89 \pm 15.49^{a, b, c}$ & $292.75 \pm 28.72^{a, b, c}$ \\
\hline VvSUC11-OE-10 & $46.83 \pm 0.13^{a, b}$ & $383.72 \pm 28.84^{a, b, c}$ & $342.39 \pm 20.31^{a}$ \\
\hline VvSUC12-OE-1 & $46.31 \pm 0.24^{a, b, c}$ & $381.39 \pm 31.07^{a, b, c}$ & $330.12 \pm 23.50^{a, b}$ \\
\hline VvSUC12-OE-2 & $45.89 \pm 0.13^{c}$ & $392.81 \pm 28.53^{a, b}$ & $341.58 \pm 35.02^{a, b}$ \\
\hline VvSUC12-OE-4 & $43.92 \pm 0.26^{\mathrm{e}}$ & $414.3 \pm 37.51^{\mathrm{a}}$ & $345.47 \pm 29.17^{a}$ \\
\hline VvSUC27-OE-4 & $47.02 \pm 0.25^{\mathrm{a}}$ & $318.39 \pm 27.07^{b, c, d}$ & $282.12 \pm 19.72^{a, b, c}$ \\
\hline VvSUC27-OE-5 & $44.74 \pm 0.33^{\mathrm{d}}$ & $322.81 \pm 22.53^{b, c, d}$ & $276.58 \pm 26.15^{a, b, c}$ \\
\hline VvSUC27-OE-6 & $46.13 \pm 0.24^{b, c}$ & $308.19 \pm 19.51^{\mathrm{c}, \mathrm{d}}$ & $270.54 \pm 21.43^{b, c}$ \\
\hline WT & $46.72 \pm 0.24^{\mathrm{a}, \mathrm{b}}$ & $268.57 \pm 16.79 \mathrm{~d}$ & $244.84 \pm 22.91^{\mathrm{C}}$ \\
\hline
\end{tabular}

Notes: Mean values and standard errors were calculated from five biological replicates. Significance was determined via comparison with data from the WT. Significance of each parameter was assessed using Student's t test (Tukey, $P<0.05)$.

2.6. VvSUC-OE Altered Leaf and Root Structures, Soluble Sugar Concentration, and Endogenous Auxin Levels in Plants Grown in Soil

The leaves and roots of 5-w-old VvSUC-OE lines planted in soil were cross-cut sectioned and analyzed by safranin $\mathrm{O} /$ fast green staining. The leaves of VvSUC-OE lines were thicker and had fewer chloroplasts than those of WT (Figure 5A). Moreover, the roots were also thicker, especially in the VvSUC12- and VvSUC27-OE lines (Figure 5B). The overexpression of SUTs resulted in enlarged xylem in the VvSUC11- and VvSUC12-OE lines, whereas the xylem area in the VvSUC27-OE lines was reduced compared with that of WT plants (Figure 5B). The soluble sugar concentration was significantly higher in the roots of VvSUC11- and VvSUC12-OE lines but lower in the VvSUC27-OE lines than those of WT (Figure 5C), which was consistent with sugar dose-dependent changes in xylem area. All the VvSUC-OE lines had lower soluble sugar concentration in their shoots than those in WT plants (Figure 5C), indicating an increased ability to transport sugar from the source leaves in the three transgenic plants compared with that of untransformed WT. Furthermore, the IAA concentration was also increased in 5-w-old VvSUC-OE lines compared with that in WT (Figure 5D). 

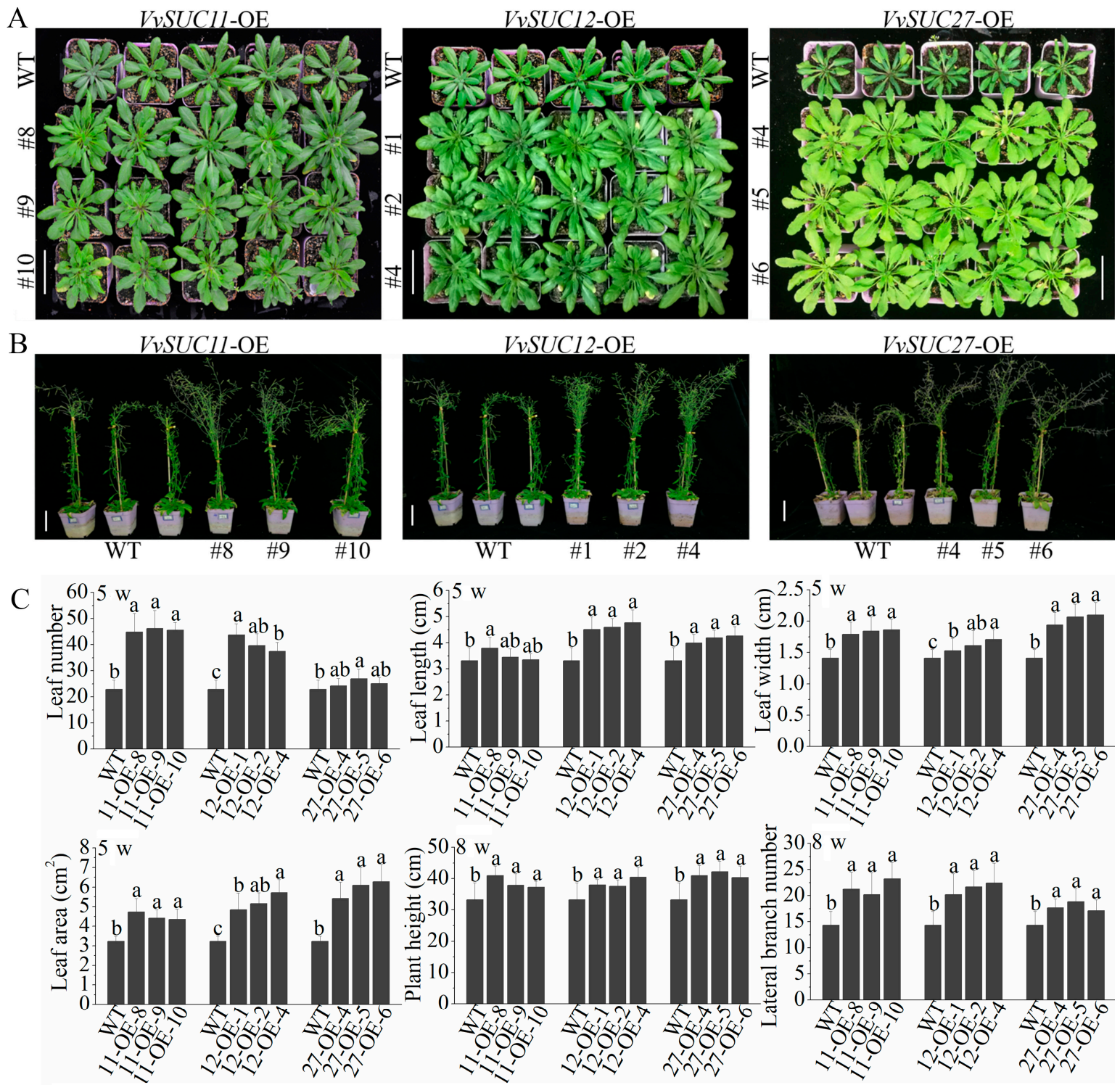

$308 \mathrm{w}$

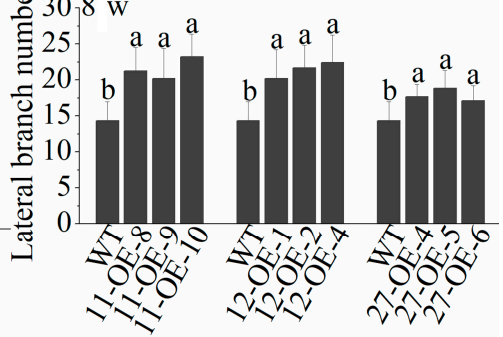

Figure 4. Phenotypes of VvSUC-OE Arabidopsis grown in soil. (A) The phenotype differences in 5-week-old VvSUC11-, VvSUC12-, and VvSUC27-OE lines, compared with those of WT. Bars $=5 \mathrm{~cm}$. (B) The phenotype differences in 8-week-old seedlings of VvSUC-OE lines and WT. Bars $=5 \mathrm{~cm}$.

(C) The phenotypic differences in leaf number, leaf length, leaf width, and leaf area between 5-week-old VvSUC-OE lines and WT and the phenotypic differences in plant height and lateral branch number between 8-week-old VvSUC-OE lines and WT. Data are expressed as mean \pm SD $(n=5)$. Different letters indicate significant differences $(P<0.05)$ between each VvSUC-OE line and WT as determined by ANOVA followed by Tukey's test. 

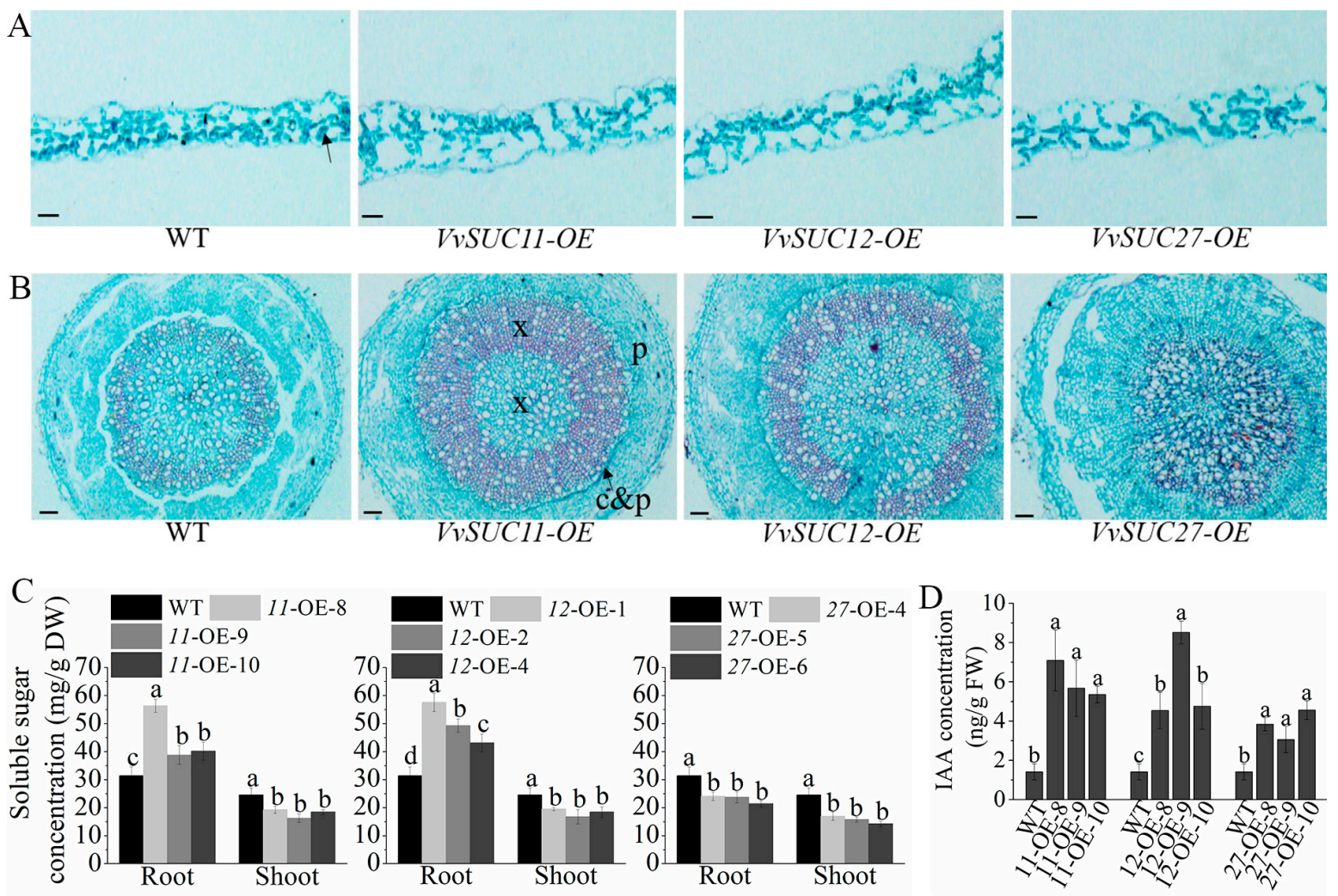

Figure 5. Internal leaf and root morphology, soluble sugar concentration, and endogenous auxin levels in 5-week-old VvSUC-OE Arabidopsis grown in soil. (A) Cross-cut leaf slices were stained with safranin $\mathrm{O} /$ fast green to visualize lignified or keratinized cell walls (red) and cytoplasm or cell walls that contain cellulose (green). Chloroplasts stained dark green and are indicated by black arrowhead. Bars $=100 \mu \mathrm{m}$. (B) Cross-cut roots slices were stained with safranin O/fast green to visualize lignified or keratinized cell wall (red) and cytoplasm or cell walls that contain cellulose (green). x, xylem; c\&p, cambium and phloem; p, periderm. Bars $=200 \mu \mathrm{m}$. (C) The soluble sugar concentrations in the roots and shoots of VvSUC-OE lines. (D) The IAA concentration in the seedlings of VvSUC-OE lines. Data are expressed as mean $\pm \mathrm{SD}(\mathrm{n}=3)$. Different letters indicate significant differences $(P<0.05)$ between each VvSUC-OE line and WT, as determined by ANOVA followed by Tukey's test.

\subsection{VvSUC-OE Lines Altered Arabidopsis Drought Resistance When Grown in Soil}

To test whether VvSUC regulate drought tolerance, VvSUC11-, VvSUC12-, and VvSUC27-OE lines together with WT were subjected to drought survival assays by withholding water for two weeks and then rewatered for another two weeks as shown in Figure 6. Before withholding water, all the VvSUC-OE lines and WT grew normally and healthily (Figure 6A). However, upon encountering drought treatment, most leaves of the plants turned yellow afterwards and were completely dried. After rewatering, the majority of VvSUC11-OE lines (excepted for VvSUC11-OE-8) (Figure 6B) and $V v S U C 27-O E$ lines (Figure 6D) rejuvenated, but nearly half of WT and nearly all the VvSUC12-OE lines (Figure 6C) did not. So compared with WT, VvSUC11 and VvSUC27 could significantly enhanced Arabidopsis drought resistance, whereas VvSUC12 could obviously decreased Arabidopsis drought resistance (Figure 6E). 

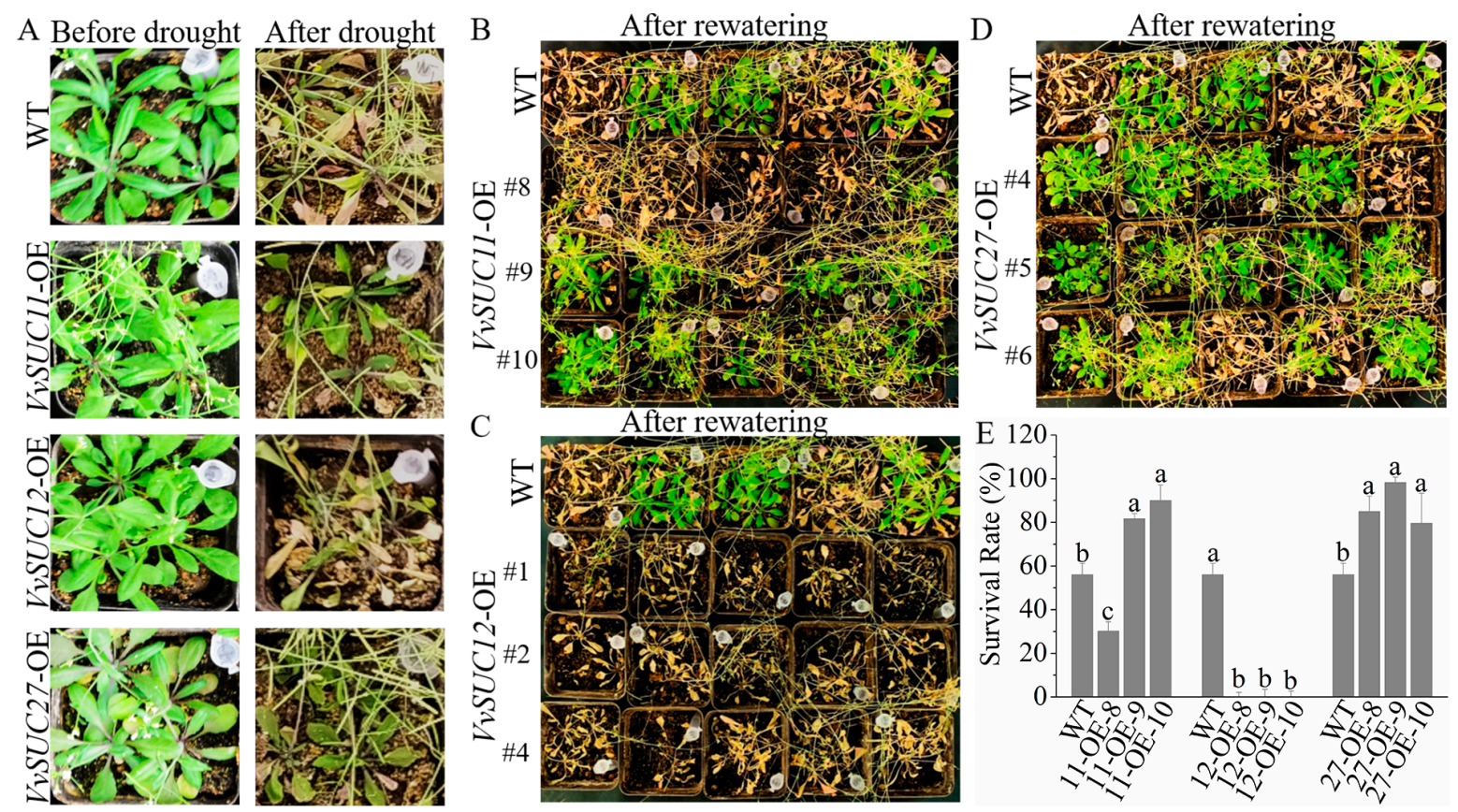

Figure 6. Phenotypic assays of VvSUC-OE Arabidopsis under drought stress. (A) Phenotypes of VvSUC-OE lines before drought and after drought for two weeks. (B) to (D) Phenotypes of (B) VvSUC11-, (C) VvSUC12-, and (D) VvSUC27-OE lines, which had survived 2 weeks after rewatering. (E) Survival rate of VvSUC-OE lines after rewatering for two weeks. The survival rates were calculated based upon three biological replicates. Each biological repeat had 20-25 seedlings (four or five seedlings for each pot) for each genotype. Data are expressed as mean \pm SD $(n=3)$. Different letters indicate significant differences $(P<0.05)$ between each VvSUC-OE line and WT, as determined by ANOVA followed by Tukey's test.

\section{Discussion}

\subsection{VvSUC11 and VvSUC12 Regulate SUT-Dependent Sucrose Transport in Similar Way}

SUT family proteins function as key sugar transporters [34]. There are three amino acids sequences encoding the formerly described sucrose transporters named VvSUC11, VvSUC12, and VvSUC27, which have been characterized [23-26]. In the phylogenetic analysis of VvSUCs, VvSUC11 was characterized as a member of the traditional low-affinity SUT4 subfamily, while VvSUC12 has 66.6\% similarity with AtSUC3 and belongs to the SUT2 subfamily (Figure S1). The members of these two subfamilies have been reported to be localized in enucleate SEs of plants [8,17]. The individual roles of VvSUC11 and VvSUC12 were further investigated in transgenic Arabidopsis overexpression lines. The phenotype and germination inhibition rate on high sucrose-supplemented media in VvSUC11-OE seeds were similar with those of WT (Figure 1), indicating that overexpression of VvSUC11 did not influence seed development. However, the germination inhibition rate in VvSUC12-OE seeds on high sucrose-supplemented media was lower than that of WT (Figure 1G). Sucrose can induce meristem quiescence as observed in the arrested development of seedlings germinated on high concentrations of sucrose [35]. Hence, VvSUC12 overexpression in Arabidopsis might have inhibited sucrose absorption by the seeds germinated on high sucrose-supplemented media, indicating a regulation role of VvSUC12 on AtSUTs as exogenous sucrose transporters.

Both VvSUC11- and VvSUC12-OE seedlings produced more and larger leaves as well as rich and thicker roots than those of WT seedlings when grown on sucrose-supplemented media (Figure 2), which resulted in a heavier dry weight of shoots and roots (Figure 3). However, the shoot/root dry weight ratio was lower in both VvSUC11- and VvSUC12-OE lines than in WT because the root proportion of the ratio increased as sucrose concentration increased. Combined with the significant 
accumulation of soluble sugar in the roots of both VvSUC11- and VvSUC12-OE, these results suggest that changes in sucrose transport alter root/shoot development as well as morphology. In higher plant species, the SUT2/SUC3-type transporter is encoded by a single gene, suggesting that SUT2/SUC3-type transporters are essential for plant growth and development. However, AtSUC3 mutants have no recognizable phenotype under standard growth conditions [14], which may act as a putative sucrose sensor in sieve elements. Although VvSUC12 belongs to SUT2/SUC3 subfamilies, we show that the situation is different from AtSUC3 mutants for VvSUC12. Furthermore, AtSUC2 has been reported to be not required for efflux in the transport and release phloem, but its retrieval function likely participates in fine-tuning whole plant carbon partitioning [36]. The changes in the proportion of sugar in the VvSUC12-OE shoots and roots were the opposite of this pattern in AtSUC2 mutants grown in sucrose-supplemented medium [37], which indicated that VvSUC12 might be a similar role in controlling carbon partitioning along the phloem path as AtSUC2. The IAA concentration increased in addition to the stronger phenotype (Figure 3C). These data suggest that VvSUC11 and VvSUC12 are high-affinity SUTs that function to fast load sucrose from the apoplast into the phloem from source when the sucrose concentration is low. This can increase IAA concentrations and encourage root development, and therefore, they can absorb sucrose from the surrounding medium, which can adversely affect photosynthesis, indicated by a decrease in chloroplasts in the leaves.

Both VvSUC11- and VvSUC12-OE lines grown in soil had similar phenotypes to when they were grown on sterile solid media, including more numbers of, larger, and thicker leaves and a higher IAA concentration than those of WT plants under the same growth conditions (Figures 4 and 5). In addition, the transformants were taller and had more lateral branches, thicker leaves, and more xylem in their roots than those of WT, which resulted in high field (Table 1). These results further verify that VvSUC11 and VvSUC12 could load sucrose produced by photosynthesis in the source leaves. The Arabidopsis AtSUC2 mutant plants grow poorly, hyperaccumulate carbohydrates in their leaves, and do not produce viable seed $[36,38]$. As a high-affinity/low-capacity (HALC) system, the SUT1 subfamily is essential for maintaining this sucrose gradient and thereby controlling the rate of phloem sucrose translocation [17]. Although VvSUC11 and VvSUC12 do not belong to the SUT1 subfamily, they are HALC transporters and their high-affinity sucrose transport is especially important for retrieval.

\subsection{VvSUC27 Shows the Strong Sucrose Transport Capacity Along the Phloem Path}

VvSUC27 is similar and belongs to the specific high-affinity SUT1 clade in dicots (Figure S1). In a previous study, we determined that VvSUC27 is a plasma membrane protein [27]. Besides, VvSUC27 was expressed in Nicotiana tabacum to produce transformants that were sensitive to sucrose and had rapid organ development, especially in roots, when cultured on sucrose-supplemented medium [27]. In this study, VvSUC27 was overexpressed in transgenic Arabidopsis to compare its function with that of the VvSUC11- and VvSUC12-OE lines. The exterior of VvSUC27-OE seeds was shorter and narrower than WT seeds, which resulted in a smaller seed area and embryo (Figure 1). We also observed the inhibition of germination on high sucrose-supplemented media compared with that of WT (Figure 1), consistent with the findings of a previous report [27]. Our results indicate that VvSUC27 has a strong sucrose transport ability that may hardly be regulated by AtSUTs or NtSUTs expressed in the seeds. The inhibitory effects on early seedling development might be due to osmotic stress, a hexokinase-independent mechanism, or changes in ABA signaling [39]. The phenotypes of VvSUC27 transgenic Arabidopsis were different from those of VvSUC27-expressing N. tabacum [27]. In this study, the VvSUC27-OE lines had inhibited root and leaf growth as well as fewer and smaller leaves; however, IAA concentration in leaves was higher than those of the WT (Figure 2), which directly resulted in lower dry weight and higher shoot/root ratio (Figure 3A). Moreover, the SUTs have the potential to form heterogenous oligomers with other SUTs in transgenic plants [40], which could also contribute to the variation in growth and development of plant varieties. The leaves of VvSUC27-OE lines turned yellow and purple on high sucrose-supplemented media and had fewer chloroplasts in the presence 
of sucrose, especially in the $6 \%$ sucrose-supplemented medium (Figure 2A). Sucrose is an effective inducer of anthocyanins in Arabidopsis [29] and AtSUC1 mutants accumulate less anthocyanins in response to exogenous sucrose [41]. Overexpression of VvSUC27 in Arabidopsis resulted in higher sugar concentration in both the shoots and roots of plants grown on $3 \%$ sucrose-supplemented medium, but lower accumulation in the roots on $6 \%$ sucrose-supplemented medium (Figure 3B), which might indicate that the roots transferred sucrose to the source organ in the presence of excess sucrose. The VvSUC27-OE lines had phenotypes similar to those of the VvSUC11- and VvSUC12-OE lines when grown in soil (Figures 4 and 5), with the exception of its reduced proportion of xylem area and an increased proportion of phloem area. In comparison to WT, these transgenic lines also had less accumulation of soluble sugar, which is consistent with the low-affinity/high capacity (LAHC) transporter, AtSUC4, which also revealed reduced sucrose concentration in overexpressing lines [42]. In contrast to HALC transporters, LAHC transporters are reported to be important for high rates of regulated phloem loading, and LAHC transport may be important under conditions where the loading rates are increased [17]. VvSUC27 as a LAHC transporter may also be important to phloem loading in vine species.

\subsection{Potential Role for SUTs in Crop Field and Drought Resistance Improvement}

Increased demand for primary foodstuffs is outstripping the increase in crop yield and therefore, plant productivity is an important goal for our society [43]. The significantly more numbers of siliques produced and the higher yield per plant (Table 1) in VvSUC-OE lines provides a promising gene target for crop improvement. Increasing photosynthetic efficiency could also improve plant productivity [44]; the $\mathrm{C}_{4}$ pathway is more efficient than $\mathrm{C}_{3}$ photosynthesis because of the higher $\mathrm{CO}_{2}$ concentration around the major $\mathrm{CO}_{2}$-fixing enzyme ribulose-1,5-bisphosphate carboxylase/oxygenase (Rubisco) [45]. Moreover, engineering traits of more efficient $C_{4}$ photosynthesis into $C_{3}$ crops could ostensibly improve water and nitrogen use efficiencies and increase plant yield in hot and dry environments [46,47]. SUTs play a key role in the allocation and partitioning of photosynthetically fixed carbon in plants and therefore, if sucrose transport efficacy is promoted, the yield of plants could be further optimized. Moreover, high light could enhance photosynthesis, leaf sugar levels, and phloem loading; however, these factors do not influence SUT expression directly [48]. Thus, SUT overexpression under high light conditions could be an important component of increased plant yield phenotypes. Here, we grew SUT-overexpressing Arabidopsis lines under high light condition to confirm that their productivity was higher than that of WT plants. We observed that the transgenic plants produced more siliques with larger size and that they were taller than WT plants (data not shown). Besides, VvSUC27-OE Arabidopsis lines could significantly enhanced Arabidopsis drought resistance (Figure 6). Most $V v S U C 11-\mathrm{OE}$ could significantly improve the drought tolerance, excepted for the VvSUC11-OE-8 line (Figure 6). Although the VvSUC11-OE lines and VvSUC12-OE lines showed the similar phonotypes in sterile and soil culture, they possess the different affinity for sucrose. VvSUC11 has a high affinity for sucrose, while VvSUC12 has a middle affinity for sucrose and its long gene contains 14 exons interrupted by 13 introns; such exon/intron organization is also described for AtSUC3 with no sucrose transport activity [23-25]. Furthermore, the subcellular localization of VvSUC11 and VvSUC12 may be different, only VvSUC11 in VvSUC has been reported to own putative dileucine-like vacuolar targeting sequence [49]. In our previous studies, SUC11 and SUC27 in resistant grapevine could fast and highly response to osmotic stress, while SUC12 nearly no response [50]. Besides affinity for sucrose, subcellular localization, and transcriptional regulation, the transport activity of sugar transporter is also affected by posttranslational modification. Drought has been reported to induce a possible posttranslational modification for SUT protein in phosphorylation [51], which might cause the different drought tolerance in VvSUC11- and VvSUC12-OE lines. This study provides a promising method to develop SUT-transgenic crops that have high productivity under high light or water shortage culture conditions, such as those common to Xin Jiang, China; Perth, Australia; and the Sahara Desert, Africa. 


\section{Materials and Methods}

\subsection{Phylogeny and Sequence Similarities Analysis}

Phylogenetic analysis of A. thaliana, L. esculentum, and V. vinifera sugar transporter protein sequences were performed using neighbor-joining method with 1000 bootstrap replication by MEGA-X (https://www.megasoftware.net/). VvSUCs sequence similarities analysis were performed by DNAMAN (https://www.lynnon.com/).

\subsection{Plant Material and Growth Conditions}

The leaves of Vitis vinifera "Thompson Seedless" variety were collected in Shangzhuang (Beijing, China). The leaves were harvested, immediately frozen in liquid nitrogen, and stored at $-80{ }^{\circ} \mathrm{C}$.

Arabidopsis thaliana ecotype Columbia (Col-0) was used in this study. Arabidopsis thaliana seeds were surface-sterilized with successive washes in $75 \%$ ethanol and $10 \% \mathrm{NaClO}$, and then rinsed 4-6 times with sterile distilled water. The sterilized seeds were sown and grew on MS media containing $0 \%, 3 \%$, or $6 \%(w / v)$ sucrose and $0.7 \%(w / v)$ agar in $640 \mathrm{~mL}$ sterile culture vessels until phenotype observation on seedlings. For Arabidopsis grown in soil, 1/2 MS medium (the half concentration of full MS medium) cultured 2-week-old seedlings were transferred to pots containing vermiculite, black soil, and nutrient soil mixture (1:1:1). All plants were place under 16-h light/8-h dark photoperiod with $3000 \mathrm{~lx}$ illuminance in growth chambers at $22^{\circ} \mathrm{C}$.

\subsection{Generation of Transgene Constructs and Plant Transformation}

Gateway-PCR was used to generate SUT-overexpressing constructs. The VvSUC11, VvSUC12, and VvSUC27 full-length coding regions were amplified from V. vinifera "Thompson Seedless" with primers that added the att sequences (attB1 and attB2 underlined inTable S2) to the $5^{\prime}$ ends of the PCR products. The primers attB1-For and attB2-Rev were used to extend the attB-adapter sequences. The attB-PCR products were mixed with the $\mathrm{pDONR} 222$ vector, $5 \times \mathrm{BP}-C_{\text {Clonase }}^{\mathrm{TM}} \mathrm{Buffer}$, and BP-Clonase ${ }^{\mathrm{TM}}$ (Invitrogen, Carlsbad, CA, USA), and incubated for $4-6 \mathrm{~h}$ at $25^{\circ} \mathrm{C}$ before proteinase $\mathrm{K}$ was added to cease the BP reaction. The entry clones were combined with the $\mathrm{pH} 7 \mathrm{WG} 2 \mathrm{D}, 1$ vector, $5 \times$ LR-Clonase ${ }^{\mathrm{TM}}$ Buffer, and LR-Clonase $^{\text {тм }}$ (Invitrogen, Carlsbad, CA, USA). The pH7WG2D,1-VvSUC11, pH7WG2D,1-VvSUC12, and pH7WG2D,1-VvSUC27 constructs were then confirmed by sequence for plant transformation and the SUTs were expressed under the strong CaMV 35S promoter in the pDR196 vector.

For Arabidopsis transformation, the SUT constructs described above were transformed into Agrobacterium tumefaciens GV3101, which was then used to transform plants using the floral dip method [52]. The seeds harvested from transformed plants were selected for homozygous $\mathrm{T}_{3}$ lines, which were used for further phenotypic analyses.

\section{4. $q R T-P C R$}

Total RNA from transgenic Arabidopsis tissues were isolated as above. Actin from Arabidopsis was used as the internal control to normalize cDNA samples. qRT-PCR was performed using the UltraSYBR Mixture Kit (CWBIO, Beijing, China) in a Rotor-Gene ${ }^{\circledR} S Y B R{ }^{\circledR}$ Green PCR (QIAGEN, Hilden, Germany) with gene-specific primers (Table S3). The threshold cycle for each qPCR with different concentrations of cDNA was determined and compared against the standards (Actin for Arabidopsis).

\subsection{Scanning Electron Microscopy}

Arabidopsis seeds were attached to conducting double-sided adhesive tape (Plano, Wetzlar, Germany). Scanning electron microscopy aluminum specimen holders were covered with the adhesive tape with seeds attached. The specimens were coated with gold for $60 \mathrm{~s}$ and observed under the FEI Quanta 200 scanning electron microscope (FEI QUANTA200, Holland). 


\subsection{Anatomical Section Analysis}

5-w-old cross-cut leaves and roots of Arabidopsis grown on soil were selected and used for safranin $\mathrm{O} /$ fast green staining, as described [53]. The lignin and fiber tissues were stained red and green, respectively, and observed under the Axio Scope A1 (ZEISS, Germany) microscope.

\subsection{Dry Weight Determination}

Four-week-old Arabidopsis plants were harvested from sterile MS media supplemented with sucrose at different concentrations. The roots and shoots (leaves and stems) of each plant were separated and dried at $80^{\circ} \mathrm{C}$ until the samples reached a constant weight.

\subsection{Soluble Sugar Measurements}

Soluble sugars were extracted from samples by grinding $5 \mathrm{~g}$ of frozen grape berries or $25 \mathrm{mg}$ of dried leaves of $A$. thaliana in $30 \mathrm{~mL}$ or $3 \mathrm{~mL}$ of $\mathrm{ddH}_{2} \mathrm{O}$, respectively, and then distilled three times at $100{ }^{\circ} \mathrm{C}$ for $30 \mathrm{~min}$. Then, $\mathrm{ddH}_{2} \mathrm{O}$ was added to the samples until the extract solutions reached a final volume of $100 \mathrm{~mL}$ for grape berries and $10 \mathrm{~mL}$ for $A$. thaliana leaves. Measurements were performed as previously described with some modifications $[54,55]$. A $0.5 \mathrm{~mL}$ aliquot of extract solution was mixed with $5 \mathrm{~mL}$ of $4 \%$ anthrone solution (dissolved in $88 \%$ concentrated sulfuric acid) and incubated in a $90{ }^{\circ} \mathrm{C}$ water bath for $15 \mathrm{~min}$. The optical density of both extracts was measured at $620 \mathrm{~nm}$.

\subsection{Determination of Endogenous Phytohormone Concentration}

Endogenous indole-3-acetic acid (IAA) was extracted from Arabidopsis seedlings grown on sterile solid media and in soil as described previously $[27,56]$ and measured using a high-performance liquid chromatography-mass chromatography system (HPLC-MS) (Varian, PaloAlto, CA, USA) in the Beijing Center for Physical and Chemical Analysis.

\subsection{Drought Stress Treatment}

For drought treatment, sterilized Arabidopsis thaliana seeds were placed on Semi-MS media as above for 2 weeks. Then the seedlings were transferred to each pot containing same weighed soil. Then the seedlings were watered once per week. After four weeks in soil, water was withheld for 2 weeks. The survival rates were calculated based upon plants that had survived 2 weeks after rewatering from three biological replicates. Each biological repeat had 20-25 seedlings ( 4 or 5 seedlings for each pot) for each genotype.

\subsection{Statistical Analyses}

One-way analysis of variance (ANOVA) and Tukey's test were performed to analyze the significance of data using SPSS16.0 (SPSS Corp., Chicago, IL, USA). We used $P<0.05$ as an indicator of statistical significance.

Supplementary Materials: Supplementary materials can be found at http://www.mdpi.com/1422-0067/21/7/2624/ s1.

Author Contributions: Data curation, Y.C., J.Y., W.T., Z.D., W.D., H.G., J.X. and N.Z.; Formal analysis, Y.Z.; Funding acquisition, L.Y., Q.M. and Y.Z.; Methodology, Y.C. and J.Y.; Project administration, Y.C., J.Y., W.T., Z.D., W.D., H.G., J.X. and Y.Z.; Resources, W.T.; Writing-original draft, Y.C.; Writing—review and editing, Y.Z. All authors have read and agreed to the published version of the manuscript.

Funding: This work was supported by the National Natural Science Foundation of China (Grant No. 30900969), the Natural Science Foundation of Guangxi (2017GXNSFBA198201), the Science and Technology Development Fund of Guangxi Academy of Agricultural Sciences (2015YT95, 2018YM11 and 2018JZ33), and the Project for Extramural Scientists of State Key Laboratory of Agrobiotechnology (2020SKLAB6-8).

Conflicts of Interest: The authors declare no conflict of interest. 


\section{Abbreviations}

$\begin{array}{ll}\text { SUT or SUC } & \text { sucrose transporter } \\ \text { SE } & \text { sieve element } \\ \text { LAHC } & \text { low-affinity/high capacity } \\ \text { OE } & \text { overexpressing } \\ \text { WT } & \text { wild type } \\ \text { MS } & \text { Murashige and Skoog } \\ \text { DW } & \text { dry weight } \\ \text { FW } & \text { fresh weight } \\ \text { x } & \text { xylem } \\ \text { c\&p } & \text { cambium and phloem } \\ \text { p } & \text { periderm } \\ \text { IAA } & \text { indole-3-acetic acid } \\ \text { qRT-PCR } & \text { quantitative real-time polymerase chain reaction }\end{array}$

\section{References}

1. Koch, K. Sucrose metabolism: Regulatory mechanisms and pivotal roles in sugar sensing and plant development. Curr. Opin. Plant Biol. 2004, 7, 235-246. [CrossRef] [PubMed]

2. Riesmeier, J.W.; Willmitzer, L.; Frommer, W.B. Isolation and characterization of a sucrose carrier cDNA from spinach by functional expression in yeast. EMBO J. 1992, 11, 4705-4713. [CrossRef] [PubMed]

3. Weise, A.; Lalonde, S.; Kuhn, C.; Frommer, W.B.; Ward, J.M. Introns control expression of sucrose transporter LeSUT1 in trichomes, companion cells and in guard cells. Plant Mol. Biol. 2008, 68, 251-262. [CrossRef] [PubMed]

4. Wang, L.F.; Qi, X.X.; Huang, X.S.; Xu, L.L.; Jin, C.; Wu, J.; Zhang, S.L. Overexpression of sucrose transporter gene PbSUT2 from Pyrus bretschneideri, enhances sucrose content in Solanum lycopersicum fruit. Plant Physiol. Biochem. 2016, 105, 150-161. [CrossRef]

5. Sun, Y.; Ward, J.M. Arg188 in rice sucrose transporter OsSUT1 is crucial for substrate transport. BMC Biochem. 2012, 13, 26. [CrossRef]

6. Scofield, G.N.; Hirose, T.; Aoki, N.; Furbank, R.T. Involvement of the sucrose transporter, OsSUT1, in the long-distance pathway for assimilate transport in rice. J. Exp. Bot. 2007, 58, 3155-3169. [CrossRef]

7. Chincinska, I.A.; Liesche, J.; Krugel, U.; Michalska, J.; Geigenberger, P.; Grimm, B.; Kuhn, C. Sucrose transporter StSUT4 from potato affects flowering, tuberization, and shade avoidance response. Plant Physiol. 2008, 146, 515-528. [CrossRef]

8. Kuhn, C.; Grof, C.P. Sucrose transporters of higher plants. Curr. Opin. Plant Biol. 2010, 13, 288-298. [CrossRef]

9. Kuhn, C.; Franceschi, V.R.; Schulz, A.; Lemoine, R.; Frommer, W.B. Macromolecular trafficking indicated by localization and turnover of sucrose transporters in enucleate sieve elements. Science 1997, 275, 1298-1300. [CrossRef]

10. Hackel, A.; Schauer, N.; Carrari, F.; Fernie, A.R.; Grimm, B.; Kuhn, C. Sucrose transporter LeSUT1 and LeSUT2 inhibition affects tomato fruit development in different ways. Plant J. 2006, 45, 180-192. [CrossRef]

11. Barker, L.; Kuhn, C.; Weise, A.; Schulz, A.; Gebhardt, C.; Hirner, B.; Hellmann, H.; Schulze, W.; Ward, J.M.; Frommer, W.B. SUT2, a putative sucrose sensor in sieve elements. Plant Cell 2000, 12, 1153-1164. [CrossRef]

12. Knop, C.; Stadler, R.; Sauer, N.; Lohaus, G. AmSUT1, a sucrose transporter in collection and transport phloem of the putative symplastic phloem loader Alonsoa meridionalis. Plant Physiol. 2004, 134, 204-214. [CrossRef]

13. Schulze, W.; Weise, A.; Frommer, W.B.; Ward, J.M. Function of the cytosolic N-terminus of sucrose transporter AtSUT2 in substrate affinity. FEBS Lett. 2000, 485, 189-194. [CrossRef]

14. Barth, I.; Meyer, S.; Sauer, N. PmSUC3: Characterization of a SUT2/SUC3-type sucrose transporter from Plantago major. Plant Cell 2003, 15, 1375-1385. [CrossRef]

15. Meyer, S.; Lauterbach, C.; Niedermeier, M.; Barth, I.; Sjolund, R.D.; Sauer, N. Wounding enhances expression of AtSUC3, a sucrose transporter from Arabidopsis sieve elements and sink tissues. Plant Physiol. 2004, 134, 684-693. [CrossRef]

16. Leach, K.A.; Tran, T.M.; Slewinski, T.L.; Meeley, R.B.; Braun, D.M. Sucrose transporter2 contributes to maize growth, development, and crop yield. J. Integr. Plant Biol. 2017, 59, 390-408. [CrossRef] 
17. Weise, A.; Barker, L.; Kuhn, C.; Lalonde, S.; Buschmann, H.; Frommer, W.B.; Ward, J.M. A new subfamily of sucrose transporters, SUT4, with low affinity/high capacity localized in enucleate sieve elements of plants. Plant Cell 2000, 12, 1345-1355. [CrossRef]

18. Chincinska, I.; Gier, K.; Krugel, U.; Liesche, J.; He, H.; Grimm, B.; Harren, F.J.; Cristescu, S.M.; Kuhn, C. Photoperiodic regulation of the sucrose transporter StSUT4 affects the expression of circadian-regulated genes and ethylene production. Front. Plant Sci. 2013, 4, 26. [CrossRef]

19. Endler, A.; Meyer, S.; Schelbert, S.; Schneider, T.; Weschke, W.; Peters, S.W.; Keller, F.; Baginsky, S.; Martinoia, E.; Schmidt, U.G. Identification of a vacuolar sucrose transporter in barley and Arabidopsis mesophyll cells by a tonoplast proteomic approach. Plant Physiol. 2006, 141, 196-207. [CrossRef]

20. Chen, Q.; Diao, L.; Song, H.; Zhu, X. Vitis amurensis Rupr: A review of chemistry and pharmacology. Phytomed. Int. J. Phytother. Phytopharm. 2018, 49, 111-122. [CrossRef]

21. Chen, J.; Wang, N.; Fang, L.C.; Liang, Z.C.; Li, S.H.; Wu, B.H. Construction of a high-density genetic map and QTLs mapping for sugars and acids in grape berries. BMC Plant Biol. 2015, 15, 28. [CrossRef]

22. Davies, C.; Wolf, T.; Robinson, S.P. Three putative sucrose transporters are differentially expressed in grapevine tissues. Plant Sci. 1999, 147, 93-100. [CrossRef]

23. Manning, K.; Davies, C.; Bowen, H.C.; White, P.J. Functional characterization of two ripening-related sucrose transporters from grape berries. Ann. Bot. 2001, 87, 125-129. [CrossRef]

24. Ageorges, A.; Issaly, R.; Picaud, S.; Delrot, S.; Romieu, C. Identification and functional expression in yeast of a grape berry sucrose carrier. Plant Physiol. Biochem. 2000, 38, 177-185. [CrossRef]

25. Afoufa-Bastien, D.; Medici, A.; Jeauffre, J.; Coutos-Thevenot, P.; Lemoine, R.; Atanassova, R.; Laloi, M. The Vitis vinifera sugar transporter gene family: Phylogenetic overview and macroarray expression profiling. BMC Plant Biol. 2010, 10, 1741-1763. [CrossRef]

26. Zhang, Y.L.; Meng, Q.Y.; Zhu, H.L.; Guo, Y.; Gao, H.Y.; Luo, Y.B.; Lu, J.A. Functional characterization of a LAHC sucrose transporter isolated from grape berries in yeast. Plant Growth Regul. 2008, 54, 71-79. [CrossRef]

27. Cai, Y.; Tu, W.; Zu, Y.; Jing, Y.; Xu, Z.; Lu, J.; Zhang, Y. Overexpression of a Grapevine Sucrose Transporter (VvSUC27) in Tobacco Improves Plant Growth Rate in the Presence of Sucrose In vitro. Front. Plant Sci. 2017, 8, 1069. [CrossRef]

28. Moore, C.R.; Gronwall, D.S.; Miller, N.D.; Spalding, E.P. Mapping quantitative trait loci affecting Arabidopsis thaliana seed morphology features extracted computationally from images. G3 2013, 3, 109-118. [CrossRef]

29. Teng, S.; Keurentjes, J.; Bentsink, L.; Koornneef, M.; Smeekens, S. Sucrose-specific induction of anthocyanin biosynthesis in Arabidopsis requires the MYB75/PAP1 gene. Plant Physiol. 2005, 139, 1840-1852. [CrossRef]

30. Shi, H.; Liu, G.; Wei, Y.; Chan, Z. The zinc-finger transcription factor ZAT6 is essential for hydrogen peroxide induction of anthocyanin synthesis in Arabidopsis. Plant Mol. Biol. 2018, 97, 165-176. [CrossRef]

31. Zhang, X.; Abrahan, C.; Colquhoun, T.A.; Liu, C.J. A Proteolytic Regulator Controlling Chalcone Synthase Stability and Flavonoid Biosynthesis in Arabidopsis. Plant Cell 2017, 29, 1157-1174. [CrossRef] [PubMed]

32. Kim, J.; Lee, W.J.; Vu, T.T.; Jeong, C.Y.; Hong, S.W.; Lee, H. High accumulation of anthocyanins via the ectopic expression of AtDFR confers significant salt stress tolerance in Brassica napus L. Plant Cell Rep. 2017, 36, 1215-1224. [CrossRef] [PubMed]

33. Liu, Y.; Shi, Z.; Maximova, S.; Payne, M.J.; Guiltinan, M.J. Proanthocyanidin synthesis in Theobroma cacao: Genes encoding anthocyanidin synthase, anthocyanidin reductase, and leucoanthocyanidin reductase. BMC Plant Biol. 2013, 13, 202. [CrossRef]

34. Jameson, P.E.; Dhandapani, P.; Novak, O.; Song, J. Cytokinins and Expression of SWEET, SUT, CWINV and AAP Genes Increase as Pea Seeds Germinate. Int. J. Mol. Sci. 2016, 17, 2013. [CrossRef]

35. Lastdrager, J.; Hanson, J.; Smeekens, S. Sugar signals and the control of plant growth and development. J. Exp. Bot. 2014, 65, 799-807. [CrossRef]

36. Srivastava, A.C.; Ganesan, S.; Ismail, I.O.; Ayre, B.G. Functional characterization of the Arabidopsis AtSUC2 Sucrose/H+ symporter by tissue-specific complementation reveals an essential role in phloem loading but not in long-distance transport. Plant Physiol. 2008, 148, 200-211. [CrossRef]

37. Gong, X.; Liu, M.; Zhang, L.; Ruan, Y.; Ding, R.; Ji, Y.; Zhang, N.; Zhang, S.; Farmer, J.; Wang, C. Arabidopsis AtSUC2 and AtSUC4, encoding sucrose transporters, are required for abiotic stress tolerance in an ABA-dependent pathway. Physiol. Plant 2015, 153, 119-136. [CrossRef] 
38. Gottwald, J.R.; Krysan, P.J.; Young, J.C.; Evert, R.F.; Sussman, M.R. Genetic evidence for the in planta role of phloem-specific plasma membrane sucrose transporters. Proc. Natl. Acad. Sci. USA 2000, 97, 13979-13984. [CrossRef]

39. Gibson, S.I. Control of plant development and gene expression by sugar signaling. Curr. Opin. Plant Biol. 2005, 8, 93-102. [CrossRef]

40. Reinders, A.; Schulze, W.; Kuhn, C.; Barker, L.; Schulz, A.; Ward, J.M.; Frommer, W.B. Protein-protein interactions between sucrose transporters of different affinities colocalized in the same enucleate sieve element. Plant Cell 2002, 14, 1567-1577. [CrossRef]

41. Sivitz, A.B.; Reinders, A.; Ward, J.M. Arabidopsis sucrose transporter AtSUC1 is important for pollen germination and sucrose-induced anthocyanin accumulation. Plant Physiol. 2008, 147, 92-100. [CrossRef]

42. Schneider, S.; Hulpke, S.; Schulz, A.; Yaron, I.; Holl, J.; Imlau, A.; Schmitt, B.; Batz, S.; Wolf, S.; Hedrich, R.; et al. Vacuoles release sucrose via tonoplast-localised SUC4-type transporters. Plant Biol. 2012, 14, 325-336. [CrossRef]

43. Long, S.P.; Marshall-Colon, A.; Zhu, X.G. Meeting the global food demand of the future by engineering crop photosynthesis and yield potential. Cell 2015, 161, 56-66. [CrossRef]

44. Schulze, S.; Westhoff, P.; Gowik, U. Glycine decarboxylase in C3, C4 and C3-C4 intermediate species. Curr. Opin. Plant Biol. 2016, 31, 29-35. [CrossRef]

45. Brautigam, A.; Gowik, U. Photorespiration connects C3 and C4 photosynthesis. J. Exp. Bot. 2016, 67, 2953-2962. [CrossRef]

46. Hibberd, J.M.; Covshoff, S. The regulation of gene expression required for C4 photosynthesis. Annu. Rev. Plant Biol. 2010, 61, 181-207. [CrossRef]

47. Wang, L.; Czedik-Eysenberg, A.; Mertz, R.A.; Si, Y.; Tohge, T.; Nunes-Nesi, A.; Arrivault, S.; Dedow, L.K.; Bryant, D.W.; Zhou, W.; et al. Comparative analyses of C(4) and C(3) photosynthesis in developing leaves of maize and rice. Nat. Biotechnol. 2014, 32, 1158-1165. [CrossRef]

48. Xu, Q.Y.; Chen, S.Y.; Ren, Y.J.; Chen, S.L.; Liesche, J. Regulation of Sucrose Transporters and Phloem Loading in Response to Environmental Cues. Plant Physiol. 2018, 176, 930-945. [CrossRef]

49. Reinders, A.; Sivitz, A.B.; Ward, J.M. Evolution of plant sucrose uptake transporters. Front. Plant Sci. 2012, 3 , 22. [CrossRef]

50. Cai, Y.; Yan, J.; Li, Q.; Deng, Z.; Liu, S.; Lu, J.; Zhang, Y. Sucrose transporters of resistant grapevine are involved in stress resistance. Plant Mol. Biol. 2019, 100, 111-132. [CrossRef]

51. Ma, Q.J.; Sun, M.H.; Lu, J.; Kang, H.; You, C.X.; Hao, Y.J. An apple sucrose transporter MdSUT2.2 is a phosphorylation target for protein kinase MdCIPK22 in response to drought. Plant Biotechnol. J. 2019, 17, 625-637. [CrossRef] [PubMed]

52. Bent, A. Arabidopsis thaliana floral dip transformation method. Methods Mol. Biol. 2006, 343, 87-103. [PubMed]

53. Wu, X.J.; Sun, S.; Xing, G.M.; Wang, G.L.; Wang, F.; Xu, Z.S.; Tian, Y.S.; Hou, X.L.; Xiong, A.S. Elevated Carbon Dioxide Altered Morphological and Anatomical Characteristics, Ascorbic Acid Accumulation, and Related Gene Expression during Taproot Development in Carrots. Front. Plant Sci. 2016, 7, 2026. [CrossRef] [PubMed]

54. Zhang, S.W.; Gan, Y.T.; Xu, B.L. Application of Plant-Growth-Promoting Fungi Trichoderma longibrachiatum T6 Enhances Tolerance of Wheat to Salt Stress through Improvement of Antioxidative Defense System and Gene Expression. Front. Plant Sci. 2016, 7, 1405. [CrossRef] [PubMed]

55. Somani, B.L.; Khanade, J.; Sinha, R. A modified anthrone-sulfuric acid method for the determination of fructose in the presence of certain proteins. Anal. Biochem. 1987, 167, 327-330. [CrossRef]

56. Pan, X.; Welti, R.; Wang, X. Quantitative analysis of major plant hormones in crude plant extracts by high-performance liquid chromatography-mass spectrometry. Nat. Protoc. 2010, 5, 986-992. [CrossRef]

(C) 2020 by the authors. Licensee MDPI, Basel, Switzerland. This article is an open access article distributed under the terms and conditions of the Creative Commons Attribution (CC BY) license (http://creativecommons.org/licenses/by/4.0/). 